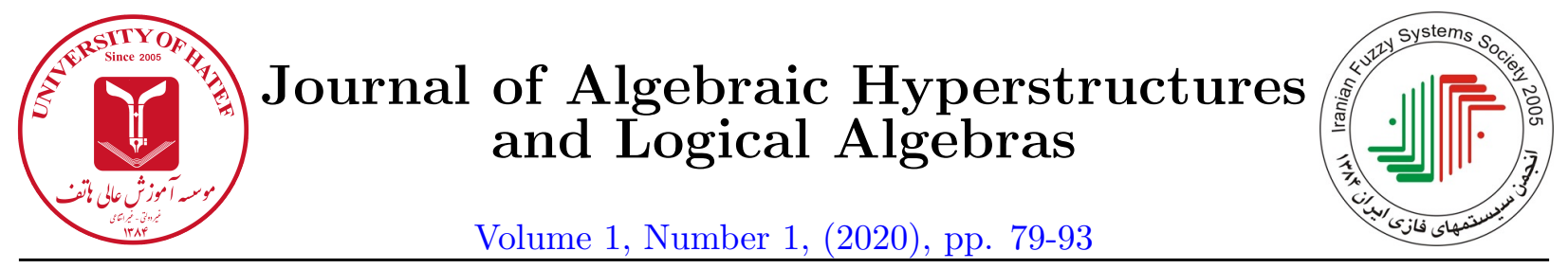

\title{
The category of hyper residuated lattices
}

\author{
O. Zahiri ${ }^{1}$ \\ ${ }^{1}$ Tehran, Iran \\ zahiri@protonmail.com
}

\begin{abstract}
In this paper, first we consider homomorphisms and also strong homomorphisms between hyper residuated lattices, and their properties are presented. Then we use strong homomorphisms to introduce the category of hyper residuated lattices. We show that this category is neither complete but not cocomplete. Moreover, we find some conditions under which the equalizers and pullbacks exist. Finally, we verify subdirectly irreducible hyper residuated lattices and attempt to construct a hyper residuated lattice from a residuated lattice.
\end{abstract}

\section{Article Information}

Corresponding Author:

O. Zahiri;

Received: December 2019;

Accepted: Invited paper.

Paper type: Original.

\section{Keywords:}

Hyper residuated lattice, category, terminal, initial.

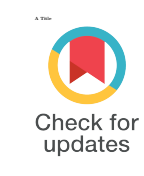

\section{Introduction}

Residuated lattices, introduced by Ward and Dilworth [13], are a common structure among algebras associated with logical systems. In this definition to any bounded lattice $(\mathcal{L}, \vee, \wedge, 0,1)$, a multiplication ' $*$ ' and an operation ' $\rightarrow$ ' are equipped such that $(\mathcal{L}, *, 1)$ is a commutative monoid and the pair $(*, \rightarrow)$ is an adjoint pair, that is,

$$
x * y \leq z \text { if and only if } x \leq y \rightarrow z, \forall x, y, z \in \mathcal{L} .
$$

The main examples of residuated lattices are $M V$-algebras introduced by Chang [6] and $B L$ algebras introduced by Hájek [11]. The hyperstructure theory was introduced by Marty [1], at the 8th Congress of Scandinavian Mathematicians. In his definition, a function $f: A \times A \longrightarrow P^{*}(A)$, of the set $A \times A$ into the set of all non-empty subsets of $A$, is called a binary hyperoperation, and the pair $(A, f)$ is called a hypergroupoid. If $f$ is associative, that is, $f(f(a, b), c)=f(a, f(b, c))$, for all $a, b, c \in A$, then $A$ is called a semihypergroup, and it is said to be commutative if $f$ is commutative, that is, $f(a, b)=f(b, a)$, for all $(a, b) \in A \times A$. Moreover, an element $1 \in A$ is said to be a unit 
or an identity element, if $a \in f(1, a)=f(1, a)$, for all $a \in A$. Many researchers have been working on this area, for examle, R. A. Borzooei et al. introduced and studied hyper $K$-algebras [5] and S. Ghorbani et al. [10, applied the hyperstructures to $M V$-algebras and introduced the concept of a hyper $M V$-algebra, which is a generalization of an $M V$-algebra. In [12, Mittas et al. applied the hyperstructures to lattices and introduced the concepts of a hyperlattice and supperlattice: A superlattice is a partially ordered set $(S ; \leq)$ endowed with two binary hyperoperations $\vee$ and $\wedge$ satisfying the following properties: for all $a, b, c \in S$,

(SL1) $a \in(a \vee a) \cap(a \wedge a)$,

(SL2) $a \vee b=b \vee a, a \wedge b=b \wedge a$,

(SL3) $(a \vee b) \vee c=a \vee(b \vee c),(a \wedge b) \wedge c=a \wedge(b \wedge c)$,

(SL4) $a \in((a \vee b) \wedge a) \cap((a \wedge b) \vee a)$,

(SL5) $a \leq b$ implies $b \in a \vee b$ and $a \in a \wedge b$,

(SL6) if $a \in a \wedge b$ or $b \in a \vee b$, then $a \leq b$.

Hyperstructures have many applications to several sectors of both pure and applied sciences. A short review of the theory of hyperstructures appear in [7]. In [8] a wealth of applications can be found, as well. There are applications to the following subjects: geometry, hypergraphs, binary relations, lattices, fuzzy set and rough sets, automata, cryptography, combinatorics, codes, artificial intelligence and probabilities. In [14], we introduced the concept of a hyper residuated lattice. We studied hyper residuated lattice congruences . In this paper, we introduce the category of hyper residuated lattices and study some categorical properties of this category. More precisely, we find products, equalizers and pullbacks in this category and also we show that the coproducts do not necessarily exist in this category.

\section{Preliminaries}

In this section, we give some preliminaries needed in the sequel.

Recall that [3, 9, 11] a residuated lattice is an algebra $(L, \vee, \wedge, \odot, \rightarrow, 0,1)$ of type $(2,2,2,2,0,0)$ such that:

(1) $(L, \vee, \wedge, 0,1)$ is a bounded lattice with 1 as the greatest element and 0 as the smallest element;

(2) $(L, \odot, 1)$ is a commutative monoid;

(3) $a \leq b \rightarrow c$ if and only if $a \odot b \leq c$, for all $a, b, c \in L$;

where for $x, y \in L, x \leq y \Leftrightarrow x=x \wedge y \Leftrightarrow y=x \vee y$. The following properties hold for any residuated lattice:

(R1) $x \leq y$ implies $z \rightarrow x \leq z \rightarrow y$ and $y \rightarrow z \leq x \rightarrow z$,

(R2) $x \leq y$ implies $x \odot z \leq y \odot z$.

Definition 2.1. [14 By a hyper residuated lattice we mean a non-empty partially ordered set $(L, \leq)$ endowed with four binary hyperoperations $\vee, \wedge, \odot, \rightarrow$ and two constants 0 and 1 satisfying the following conditions:

(HRL1) $(L ; \leq, \vee, \wedge, 0,1)$ is a bounded superlattice;

(HRL2) $(L ; \odot, 1)$ is a commutative semihypergroup with 1 as the identity;

(HRL3) $a \odot c \ll b$ if and only if $c \ll a \rightarrow b$;

where $A \ll B$ means that there exist $a \in A$ and $b \in B$ such that $a \leq b$, for all non-empty subsets $A$ and $B$ of $L$. Moreover, $L$ is called non-trivial if $0 \neq 1$. An element $a \in L$ is called scalar if $|a \odot x|=1$, for each $x \in L$. 
Let $(L ; \vee, \wedge, \odot, \rightarrow, 0,1)$ be a hyper residuated lattice and $x, y \in L$ such that $x \leq y$. By (HRL2), we have $x \in 1 \odot x$ and so $1 \odot x \ll y$. Hence by (HRL3), $1 \ll x \rightarrow y$ and so $1 \in x \rightarrow y$. Hence, for all $x, y \in L$,

(P1) $x \leq y$ implies $1 \in x \rightarrow y$

Definition 2.2. 14] Let $(L ; \vee, \wedge, \odot, \rightarrow, 0,1)$ and $\left(L^{\prime} ; \vee^{\prime}, \wedge^{\prime}, \odot^{\prime}, \rightarrow^{\prime}, 0^{\prime}, 1^{\prime}\right)$ be two hyper residuated lattices and $f: L \rightarrow L^{\prime}$ be a function. Then $f$ is called a homomorphism if it satisfies the following conditions: for any $* \in\{\vee, \wedge, \odot, \rightarrow\}$ and $x, y \in L$, (i) $f(x * y) \subseteq f(x) *^{\prime} f(y)$, (ii) $f(1)=1^{\prime}$ and $f(0)=0^{\prime}$.

If $f$ satisfies (ii) and in the condition (i), the inclusion relation replaced by the equality, then $f$ is said to be a strong homomorphism (S-homomorphism, for short). A homomorphism which is one to one, onto or both is called a monomorphism, epimorphism or an isomorphism, respectively. Similarly, an $S$-homomorphism which is one-to-one, onto or both is called an S-monomorphism, S-epimorphism or S-isomorphism, respectively.

Definition 2.3. 14] Let $F$ be a non-empty subset of a hyper residuated lattice $L$ satisfying

(F) $x \leq y$ and $x \in F$ imply $y \in F$.

Then $F$ is called:

- $a$ filter if $x \odot y \subseteq F$ for all $x, y \in F$;

- a weak filter if $F \ll x \odot y$, for all $x, y \in F$.

A filter $F$ is said to be proper, if $F \neq L$, which is equivalent to $0 \notin F$. Clearly, each filter is a weak filter. Moreover, $1 \in F$, for each (weak) filter $F$ of $L$.

Definition 2.4. [1] In each category $C$, given parallel arrows $f, g: A \rightrightarrows B$

(i) an equalizer of $f$ and $g$ consists of an object $E$ and arrow $e: E \rightarrow A$ satisfying $f \circ e=g \circ e$ with a universal property, that is, for each arrow $z: Z \rightarrow A$ with $f \circ z=g \circ z$ there exists a unique arrow $u: Z \rightarrow E$ such that $e \circ u=z$.

(ii) an coequalizer of $f$ and $g$ consists of an object $Q$ and arrow $q: B \rightarrow Q$ satisfying $q \circ f=q \circ g$ with a universal property, that is, for each object $Z$ and arrow $z: B \rightarrow Z$, if $z \circ f=z \circ g$, then there exists a unique $u: Q \rightarrow Z$ such that $u \circ q=z$.

Definition 2.5. 14] Let $L$ be a set, $\theta$ a relation on $L$ and $A, B$ two subsets of $L$. Then we write

(i) $A \theta B$ if there exist $a \in A$ and $b \in B$ such that $a \theta b$.

(ii) $A \bar{\theta} B$ if for all $a \in A$, there exists $b \in B$ such that $a \theta b$ and for all $b \in B$, there exists $a \in A$ such that $a \theta$ b.

Definition 2.6. [14] An equivalence relation $\theta$ on a hyper residuated lattice $L$ is called a congruence relation if for all $x, y, z, w \in L, x \theta$ and $z \theta w$ imply $x * z \bar{\theta} y * w$, where $* \in\{\wedge, \vee, \odot, \rightarrow\}$.

Definition 2.7. 2] Let $\gamma$ be an equivalence relation on the partially ordered set $(P, \leq)$.

(i) By a $\gamma$-fence we shall mean an ordered subset of $P$ having the following diagram (Figure 1), where $a_{i} \leq b_{i+1}$ and three vertical lines indicate the equivalence modulo $\gamma$. We often denote this $\gamma$-fence by $\left\langle a_{1}, b_{n}\right\rangle_{\gamma}$ and say that a $\gamma$-fence $\left\langle a_{1}, b_{n}\right\rangle_{\gamma}$ joins $a_{1}$ to $b_{n}$.

(ii) By a $\gamma$-crown we shall mean an ordered subset of $P$ having the following diagram (Figure 2) 


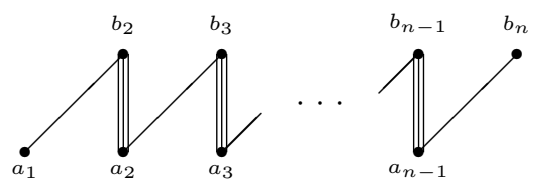

Figure 1: $\gamma$-fence

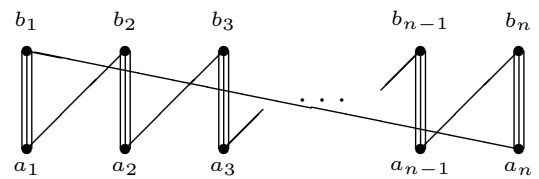

Figure 2: $\gamma$-crown

where $a_{i} \leq b_{i+1}, a_{n} \leq b_{1}$ and three vertical lines indicate the equivalence modulo $\gamma$. We often denote this $\gamma$-crown by $\left\langle\left\langle a_{1}, b_{n}\right\rangle\right\rangle_{\gamma}$.

(iii) A $\gamma$-crown $\left\langle a_{1}, b_{n}\right\rangle_{\gamma}$ is called $\gamma$-closed, when $a_{i} \gamma b_{j}$, for all $i, j \in\{1,2, \cdots, n\}$.

Definition 2.8. 2] Let $(P, \leq)$ be a partially ordered set and $\gamma$ be an equivalence relation on $P$. Then $\gamma$ is called regular if the set $P / \gamma=\{[x] \mid x \in P\}$ can be ordered in such a way that the natural map $\pi: P \rightarrow P / \gamma$ is order preserving.

Theorem 2.9. [2] Let $\gamma$ be an equivalence relation on the partially ordered set $(P, \leq)$ and $\leq_{\gamma}$ be the relation on $P / \gamma=\{[x] \mid x \in P\}$ which is defined by $[x] \leq_{\gamma}[y]$ if and only if there is a $\gamma$-fence that joins $x$ to $y$. Then the following statements are equivalent:

(i) $\leq_{\gamma}$ is an order on $P / \gamma$;

(ii) $\gamma$ is regular;

(iii) every $\gamma$-crown is $\gamma$-closed.

Definition 2.10. [14 Let $\theta$ be a regular congruence relation on a hyper residuated lattice $L$. We say that $\leq_{\theta}, \bar{\nabla}$ and $\bar{\wedge}$ are compatible if they satisfy the following conditions: for all $x, y \in L$,

(i) $[y] \in[x] \nabla[y]$ if and only if $[x] \leq_{\theta}[y]$;

(ii) $[x] \in[x] \pi[y]$ if and only if $[x] \leq_{\theta}[y]$.

By a regular compatible congruence relation on the residuated lattice $L$ we mean a regular congruence relation on $L$ such that $\leq_{\theta}, \bar{\nabla}$ and $\bar{\lambda}$ are compatible.

Theorem 2.11. [14] Let $\theta$ be a regular compatible congruence relation on a hyper residuated lattice $L$. For all $x, y \in L$, define $[x] \odot[y]=[x \odot y],[x] \nabla[y]=[x \vee y],[x] \wedge[y]=[x \wedge y]$ and $[x] \rightsquigarrow[y]=[x \rightarrow y]$, where $[A]=\{[a] \mid a \in A\}$, for all $A \subseteq L$. Then $(L / \theta ; \bar{\nabla}, \bar{\wedge}, \odot, \rightsquigarrow,[0],[1])$ is a hyper residuated lattice.

Theorem 2.12. 14 Let $(L ; \vee, \wedge, \odot, \rightarrow, 0,1)$ and $\left(L^{\prime} ; \vee^{\prime}, \wedge^{\prime}, \odot^{\prime}, \rightarrow^{\prime}, 0^{\prime}, 1^{\prime}\right)$ be two hyper residuated lattices, $f: L \rightarrow L^{\prime}$ be an $S$-homomorphism and $\operatorname{ker}(f)=\{(x, y) \in L \times L \mid f(x)=f(y)\}$. If $f(x) \leq f(y)$ implies there is a $\operatorname{ker}(f)$-fence that joins $x$ to $y$, for all $x, y \in L$, then

(i) $\operatorname{ker}(f)$ is a regular compatible congruence relation on $L$ and $L / \operatorname{ker}(f)$ is a hyper residuated lattice.

(ii) $f$ induces a unique $S$-homomorphism $\bar{f}: L / \operatorname{ker}(f) \rightarrow L^{\prime}$ by $\bar{f}([x])=f(x)$, for all $x \in L$ such that $\operatorname{Im}(\bar{f})=\operatorname{Im}(f)$ and $\bar{f}$ is an $S$-monomorphism. 


\section{$3 \quad$ Morphisms between hyper residuated lattices}

In this section, we verify the properties of homomorphisms and $S$-homomorphisms between hyper residuated lattices. After that, the relations between homomorphisms, filters and deductive systems will been studied. In the next section, using $S$-homomorphisms to introduce the category of hyper residuated lattices.

From now on, in this paper, $(L ; \vee, \wedge, \odot, \rightarrow, 0,1)$ and $\left(L^{\prime} ; \vee, \wedge, \odot, \rightarrow, 0,1\right)$ are two hyper residuated lattices, unless otherwise stated.

Note that, for a homomorphism $f: L \rightarrow L^{\prime}$ and $x, y \in L, x \leq y$ implies $f(x) \leq f(y)$. In fact, $x \leq y$ gives $x \in x \wedge y$ and so $f(x) \in f(x \wedge y) \subseteq f(x) \wedge f(y)$. Hence $f(x) \leq f(y)$, as required.

Definition 3.1. [4] A non-empty subset $D$ of a hyper residuated lattice $L$ containing 1 is called:

- A deductive system, if for all $x, y \in L, x \in D$ and $x \rightarrow y \subseteq D$ imply $y \in D$.

- A weak deductive system, if for all $x, y \in L, x \in D$ and $D \ll x \rightarrow y$ imply $y \in D$.

Notice that, for $A, B \subseteq L, A \ll B$ means that there are $a \in A$ and $b \in B$ such that $a \leq b$.

Clearly, every weak deductive system is a deductive system. A (weak) deductive system $D$ is said to be proper if $D \neq L$.

Example 3.2. Let $\left(L=L^{\prime}=\{0, a, b, 1\}, \leq\right)$ be a chain such that $0<a<b<1$. Define two hyperoperations $\vee$ and $\wedge$ on $L$ by the following tables:

Table 1

\begin{tabular}{|c|cccr|}
\hline$\vee$ & 0 & $a$ & $b$ & 1 \\
\hline 0 & $\{0, a, b, 1\}$ & $\{a, b, 1\}$ & $\{b, 1\}$ & $\{1\}$ \\
$a$ & $\{a, b, 1\}$ & $\{a, 1, b\}$ & $\{b, 1\}$ & $\{1\}$ \\
$b$ & $\{b, 1\}$ & $\{b, 1\}$ & $\{b, 1\}$ & $\{1\}$ \\
1 & $\{1\}$ & $\{1\}$ & $\{1\}$ & $\{1\}$ \\
\hline
\end{tabular}

Table 2

\begin{tabular}{|c|cccr|}
\hline$\wedge$ & 0 & $a$ & $b$ & 1 \\
\hline 0 & $\{0\}$ & $\{0\}$ & $\{0\}$ & $\{0\}$ \\
$a$ & $\{0\}$ & $\{0, a\}$ & $\{0, a\}$ & $\{0, a\}$ \\
$b$ & $\{0\}$ & $\{0, a\}$ & $\{0, b, a\}$ & $\{0, b, a\}$ \\
1 & $\{0\}$ & $\{0, a\}$ & $\{0, b, a\}$ & $\{0, a, b, 1\}$ \\
\hline
\end{tabular}

Let $x \odot y=x \wedge y$, for any $x, y \in L$ and $\odot^{\prime}: L \times L \rightarrow P^{*}(L)$ be the binary hyperoperation on $L$, which is defined by $x \odot^{\prime} y=\{\min \{x, y\}\}$, for all $x, y \in L$. Consider the following tables:

Table 3
\begin{tabular}{|c|cccr|}
\hline$\rightarrow$ & 0 & $a$ & $b$ & 1 \\
\hline 0 & $\{1\}$ & $\{1\}$ & $\{1\}$ & $\{1\}$ \\
$a$ & $\{0\}$ & $\{1, a\}$ & $\{1\}$ & $\{1\}$ \\
$b$ & $\{0\}$ & $\{a\}$ & $\{b, 1\}$ & $\{1\}$ \\
1 & $\{0\}$ & $\{a\}$ & $\{a, b\}$ & $\{1\}$ \\
\hline
\end{tabular}

Table 4
\begin{tabular}{|l|cccr|}
\hline$\rightsquigarrow$ & 0 & $a$ & $b$ & 1 \\
\hline 0 & $\{1\}$ & $\{1, b\}$ & $\{1, b\}$ & $\{1, b\}$ \\
$a$ & $\{0, a, 1\}$ & $\{1, a\}$ & $\{1\}$ & $\{1\}$ \\
$b$ & $\{0,1, a\}$ & $\{a\}$ & $\{1, b\}$ & $\{1, b\}$ \\
1 & $\{0,1\}$ & $\{1, a\}$ & $\{a, 1, b\}$ & $\{1\}$ \\
\hline
\end{tabular}

Routine calculation shows that $\left(L ; \vee, \wedge, \odot^{\prime}, \rightarrow, 0,1\right)$ and $\left(L^{\prime} ; \vee, \wedge, \odot, \rightsquigarrow, 0,1\right)$ are two hyper residuated lattices. Since $1 \rightarrow a=\{1, a\}, 1 \rightarrow b=\{1, a, b\}$ and $1 \rightarrow 0=\{0,1\}, D_{1}=\{1\}$ is a deductive system of $L^{\prime}$ but it is not a weak deductive system of $L^{\prime}$. Similarly, we can show that $D_{2}=\{1, a, b\}$ is a deductive system of $L^{\prime}$, but it is not a weak deductive system. Moreover, $D_{3}=\{1, b\}$ is a weak deductive system of $L$.

Now, we recall from [4], the following proposition needed in the sequel.

Proposition 3.3. [4 Let $L$ be a hyper residuated lattice.

(i) Every weak deductive system of $L$ satisfies $(F)$. 
(ii) Let $D$ be a non-empty subset of $L$ satisfying $(F)$. Then $D$ is a weak deductive system of $L$ if and only if $(x \rightarrow y) \cap D \neq \emptyset$ and $x \in D$ imply $y \in D$.

Theorem 3.4. Let $f: L \rightarrow L^{\prime}$ be a homomorphism between the hyper residuated lattices.

(i) If $F$ is a filter of $L^{\prime}$, then $f^{-1}(F)$ is a filter of $L$,

(ii) If $f$ is an $S$-homomorphism and $F$ is a weak filter of $L^{\prime}$, then $f^{-1}(F)$ is a weak filter of $L$. Particularly, $f^{-1}(1)$ is a weak filter of $L$,

(iii) If $f$ is an $S$-epimorphism and $F$ is a filter of $L$ containing $f^{-1}(1)$, then $f(F)$ is a filter of $L^{\prime}$

(iv) If $f$ is an $S$-epimorphism and $D$ is a weak deductive system of $L$ containing $f^{-1}(1)$, then $f(D)$ is a weak deductive system of $L^{\prime}$,

(v) If $D$ is a weak deductive system of $L^{\prime}$, then $f^{-1}(D)$ is a weak deductive system of $L$,

(vi) If $f$ is an $S$-homomorphism and $1 \odot 1=\{1\}$, then $f^{-1}(1)$ is a weak deductive system of $L$.

Proof. (i) Since $f$ is a homomorphism, $f(1)=1$ and so $1 \in f^{-1}(F)$. This gives $f^{-1}(F) \neq \emptyset$. Now, let $x \in f^{-1}(F)$ and $x \leq y$, for some $y \in L$. Then $f(x) \leq f(y)$ and $f(x) \in F$ and so $f(y) \in F$. This means that $y \in f^{-1}(F)$. Now, let $x, y \in f^{-1}(F)$. Then $f(x), f(y) \in F$ and so $f(x \odot y) \subseteq f(x) \odot f(y) \subseteq F$, hence $x \odot y \subseteq f^{-1}(F)$. Therefore, $f^{-1}(F)$ is a filter of $L$.

(ii) Similar to the proof of (i), we can show that $f^{-1}(F)$ satisfies $(F)$. Now, let $x, y \in f^{-1}(F)$. This means that $f(x), f(y) \in F$ and so $(f(x) \odot f(y)) \cap F \neq \emptyset$. Since $f$ is an $S$-homomorphism, $f(x \odot y) \cap F \neq \emptyset$ and so $(x \odot y) \cap f^{-1}(F) \neq \emptyset$. Therefore, $f^{-1}(F)$ is a weak filter of $L$.

To prove the last part of (ii), taking $F=\{1\}$. Thus $f^{-1}(1)$ is a weak filter of $L$.

(iii) By hypothesis $1 \in f(F)$ and so $f(F) \neq \emptyset$. Now, let $y \in f(F)$ and $y \leq y^{\prime}$, for some $y^{\prime} \in L^{\prime}$. This gives $y=f(x)$, for some $x \in F$. Since $f$ is onto, there exists $x^{\prime} \in L$ with $f\left(x^{\prime}\right)=y^{\prime}$. By $(P 1)$, $f(1)=1 \in f(x) \rightarrow f\left(x^{\prime}\right)=f\left(x \rightarrow x^{\prime}\right)$ thus $\left(x \rightarrow x^{\prime}\right) \cap f^{-1}(1) \neq \emptyset$. Hence there exists $u \in x \rightarrow x^{\prime}$ such that $u \in f^{-1}(1) \subseteq F$. From $u, x \in F$ and $u \in x \rightarrow x^{\prime}$ it follows that $u \odot x \subseteq F$ and $u \odot x \ll x^{\prime}$ we get that $x^{\prime} \in F$ and so $y^{\prime}=f\left(x^{\prime}\right) \in f(F)$. Now, let $u, v \in f(F)$. Then there exist $a, b \in F$ such that $f(a)=u$ and $f(b)=v$. Since $f$ is an $S$-homomorphism, $u \odot v=f(a) \odot f(b)=f(a \odot b) \subseteq f(F)$. Therefore, $f(F)$ is a filter of $L^{\prime}$.

(iv) By hypothesis $1 \in f(D)$. Let $y \in f(D)$ and $f(D) \ll y \rightarrow y^{\prime}$, for some $y^{\prime} \in L^{\prime}$. Then there are $x \in D$ and $x^{\prime} \in L$ such that $f(x)=y$ and $f\left(x^{\prime}\right)=y^{\prime}$. Since $f$ is an $S$-homomorphism, we have $f(D) \ll f\left(x \rightarrow x^{\prime}\right)$. Hence there exist $a \in D$ and $b \in x \rightarrow x^{\prime}$ such that $f(a) \leq f(b)$. By $(P 1)$, $f(1)=1 \in f(a) \rightarrow f(b)=f(a \rightarrow b)$. Thus, $(a \rightarrow b) \cap f^{-1}(1) \neq \emptyset$ and so $(a \rightarrow b) \cap D \neq \emptyset$. Since $D$ is a weak deductive system of $L$ and $a \in D, b \in D$, we get that $\left(x \rightarrow x^{\prime}\right) \cap D \neq \emptyset$. On the other hand, $D$ is a weak deductive system of $L$ and $x \in D$. Hence $x^{\prime} \in D$ and so $f\left(x^{\prime}\right) \in f(D)$. Therefore, $f(D)$ is a weak deductive system of $L^{\prime}$.

(v) Clearly, $1 \in f^{-1}(D)$. Now, let $x \in f^{-1}(D)$ and $f^{-1}(D) \ll x \rightarrow y$, for some $y \in L$. Then $f(x) \in D$ and there exists $a \in f^{-1}(D)$ such that $a \ll x \rightarrow y$. So $f(a) \in D$ and $f(a) \ll f(x \rightarrow y)$ (since $f$ is a homomorphism). Hence $D \ll f(x \rightarrow y)$. Since $f$ is a homomorphism, we have $D \ll f(x) \rightarrow f(y)$ and so $f(y) \in D$. Therefore, $y \in f^{-1}(D)$, which implies $f^{-1}(D)$ is a weak deductive system of $L$. 
(vi) Clearly, $1 \in f^{-1}(1)$. Let $x \in f^{-1}(1)$ and $f^{-1}(1) \ll x \rightarrow y$, for some $y \in L$. Then there exists $a \in f^{-1}(1)$ and $b \in x \rightarrow y$ such that $a \leq b$ and so by $(P 1), 1=f(a) \leq f(b) \in f(x \rightarrow y)$. Since $f$ is an $S$-homomorphism of $L$, then $1 \ll f(x \rightarrow y)=f(x) \rightarrow f(y)$ and so $1=1 \odot 1=$ $1 \odot f(x) \leq f(y)$. Thus, $f(y)=1$. Therefore, $f^{-1}(1)$ is a weak deductive system of $L$.

Example 3.5. Let $\left(L ; \vee, \wedge, \odot^{\prime}, \rightarrow, 0,1\right)$ and $\left(L^{\prime} ; \vee, \wedge, \odot, \rightsquigarrow, 0,1\right)$ be the hyper residuated lattices, which were defined in Example 3.2. We define $f: L \rightarrow L^{\prime}$ by $f(x)=x$, for all $x \in\{0, a, 1\}$ and $f(b)=c$. Then $f$ is an $S$-homomorphism.

Definition 3.6. Let $D$ be a proper (weak) deductive system of the hyper residuated lattice $L . D$ is said to be maximal if $D \subseteq J \subseteq L$, then $D=J$ or $J=L$, for all (weak) deductive system $J$ of $L$.

In Example 3.2, $D=\{1, a, b\}$ is a maximal (weak) deductive system of $\left(L, \vee, \wedge, \odot{ }^{\prime}, \rightarrow, 0,1\right)$. Moreover, in Example 3.2, $\{1, a, b\}$ is a maximal deductive system of $(L, \vee, \wedge, \odot, \rightsquigarrow, 0,1)$, but it is not a maximal weak deductive system of $(L, \vee, \wedge, \odot, \rightsquigarrow, 0,1)$ (since $\{1, a, b\} \ll 1 \rightarrow 0=\{1,0\}$ and $0 \notin\{1, a, b\})$.

Theorem 3.7. Let $f: L \rightarrow L^{\prime}$ be an $S$-epimorphism between the hyper residuated lattices.

(i) If $D$ is a maximal weak deductive system of $L^{\prime}$, then $f^{-1}(D)$ is a maximal weak deductive system of $L$.

(ii) If $D$ is a maximal weak deductive system of $L$ such that $f^{-1}(1) \subseteq D$, then $f(D)$ is a maximal weak deductive system of $L^{\prime}$.

(iii) If $F$ is a maximal filter of $L^{\prime}$, then $f^{-1}(F)$ is a maximal filter of $L$.

Proof. (i) By Theorem 3.4(v), $f^{-1}(D)$ is a weak deductive system of $L$. If $0 \in f^{-1}(D)$, then $0^{\prime}=f(0) \in D$ and so by Proposition 3.3(i), $D=L^{\prime}$, which is impossible. Hence $f^{-1}(D)$ is a proper weak deductive system of $L$. Now, let $J$ be a weak deductive system of $L$ such that $f^{-1}(D) \subseteq J \subseteq L$. Let $J \neq L$. Since $f^{-1}(1) \subseteq J$, by Theorem 3.4(iv), $f(J)$ is a weak deductive system of $L^{\prime}$. We show that $f(J) \neq L^{\prime}$. If $L^{\prime}=f(J)$, then $0 \in f(J)$ and so there exists $a \in J$ such that $f(a)=0$. Hence $f(a)=f(0)$. Since $f$ is an $S$-epimorphism, we get $1 \in f(a) \rightarrow f(0)=f(a \rightarrow 0)$ and so $(a \rightarrow 0) \cap f^{-1}(1) \neq \emptyset$. Moreover, $f^{-1}(1) \subseteq D \subseteq f(J)$. Since $a \in D$ and $D$ is a weak deductive system of $L$, we have $0 \in D$, which is a contradiction. Hence $f(J)$ is a proper weak deductive system of $L^{\prime}$. Thus, by $D \subseteq f\left(f^{-1}(D)\right) \subseteq f(J) \neq L^{\prime}$, we conclude that $D=f(J)$ and so $J \subseteq f^{-1}(f(J))=f^{-1}(D) \subseteq J$ implies $f^{-1}(D)=J$. Therefore, $f^{-1}(D)$ is a weak maximal deductive system of $L$.

(ii) By Theorem 3.4(iv), $f(D)$ is a weak deductive system of $L^{\prime}$ and similar to the proof of (i), we can deduce that $f(D)$ is proper. Now, let $f(D) \subseteq J \subseteq L^{\prime}$, for some weak deductive systems $J$ of $L^{\prime}$. If $J \neq L^{\prime}$, then by Theorem 3.4(v), $f^{-1}(J)$ is a weak deductive system of $L$. Moreover, $0 \notin f^{-1}(J)$. On the contrary, if $0 \in f^{-1}(J)$, then $0=f(0) \in J$, which is a contradiction. It follows that, $f^{-1}(J)$ is a proper weak deductive system of $L$. Hence $D \subseteq f^{-1}(f(D)) \subseteq f^{-1}(J)$ implies $D=f^{-1}(J)$ and so $J=f\left(f^{-1}(J)=f(D)\right.$. Therefore, $f(J)$ is a maximal weak deductive system of $L^{\prime}$.

(iii) By Theorem 3.4(i), $f^{-1}(F)$ is a filter of $L$. Similar to the proof of (i), it can be proved that $f^{-1}(F)$ is also a proper filter of $L$. Now, let $M$ be a filter of $L$ such that $f^{-1}(F) \subseteq M \subseteq L$. Let $M \neq L$. Since $f(1) \in f^{-1}(F)$, by Theorem 3.4(iii), $F=f\left(f^{-1}(F)\right)$ and $f(M)$ are filters of $L^{\prime}$. Since 
$F \subseteq f(M) \subseteq L^{\prime}$ and $F$ is a maximal filter of $L$, we get that $F=f(M)$ or $f(M)=L^{\prime}$. If $f(M)=L^{\prime}$, then $L=f^{-1}(f(M)) \subseteq M$, which is impossible. If $f(M)=F$, then $f^{-1}(F)=f^{-1}(f(M))$. Therefore, $f^{-1}(F)$ is a maximal filter of $L$.

Let $\left\{\left(L_{i} ; \vee_{i}, \wedge_{i}, \odot_{i}, \rightarrow_{i}, 0_{i}, 1_{i}\right) \mid i \in I\right\}$ be a family of the hyper residuated lattices. We define the hyperoperations $\vee, \wedge, \rightarrow$ and $\odot$ on $\prod_{i \in I} L_{i}$ as follows:

$$
\begin{aligned}
\left(x_{i}\right)_{i \in I} \vee\left(y_{i}\right)_{i \in I}=\left(x_{i} \vee_{i} y_{i}\right)_{i \in I}, & \left(x_{i}\right)_{i \in I} \wedge\left(y_{i}\right)_{i \in I}=\left(x_{i} \wedge_{i} y_{i}\right)_{i \in I} \\
\left(x_{i}\right)_{i \in I} \odot\left(y_{i}\right)_{i \in I}=\left(x_{i} \odot_{i} y_{i}\right)_{i \in I}, & \left(x_{i}\right)_{i \in I} \rightarrow\left(y_{i}\right)_{i \in I}=\left(x_{i} \rightarrow_{i} y_{i}\right)_{i \in I}
\end{aligned}
$$

where $\left(A_{i}\right)_{i \in I}=\left\{\left(a_{i}\right)_{i} \mid a_{i} \in A_{i}\right.$, for any $\left.i \in I\right\}$ and $\left(x_{i} *_{i} y_{i}\right)_{i \in I}=\left\{\left(u_{i}\right)_{i \in I} \mid u_{i} \in x_{i} *_{i} y_{i}, \forall i \in I\right\}$ for any $* \in\{\odot,, \vee, \wedge\}$, any subset $A_{i}$ of $L_{i}$ and $i \in I$. It is easy to verify that $\left(\Pi_{i \in I} L_{i}, \leq\right)$ satisfies (SL1)-(SL6) in which the order $\leq$ is given by

$$
\left(a_{i}\right)_{i \in I} \leq\left(b_{i}\right)_{i \in I} \Leftrightarrow a_{i} \leq b_{i}, \forall i \in I .
$$

Then $\left(\Pi_{i \in I} L_{i}, \leq\right)$ is a bounded supperlattice. Moreover, it is obvious that $\left(\Pi_{i \in I} L_{i}, \odot,\left(1_{i}\right)_{i \in I}\right)$ is a commutative semihypergroup with the unit $\left(1_{i}\right)_{i \in I}$. Now, if $\left(x_{i}\right)_{i \in I},\left(y_{i}\right)_{i \in I},\left(z_{i}\right)_{i \in I} \in \Pi_{i \in I} L_{i}$, then

$$
\begin{aligned}
\left(x_{i}\right)_{i \in I} \odot\left(y_{i}\right)_{i \in I} \ll\left(z_{i}\right)_{i \in I} & \Leftrightarrow\left(x_{i} \odot_{i} y_{i}\right)_{i \in I} \ll\left(z_{i}\right)_{i \in I} \\
& \Leftrightarrow\left((\forall i \in I) \quad\left(\exists u_{i} \in x_{i} \odot_{i} y_{i}\right) \quad\left(u_{i}\right)_{i \in I} \leq\left(z_{i}\right)_{i \in I}\right), \\
& \Leftrightarrow u_{i} \leq z_{i} \text {, for any } i \in I \\
& \Leftrightarrow x_{i} \odot_{i} y_{i} \ll z_{i} \text {, for any } i \in I \\
& \Leftrightarrow x_{i} \ll y_{i} \rightarrow_{i} z_{i} \text {, for any } i \in I \\
& \Leftrightarrow x_{i} \leq a_{i} \text {, for some } a_{i} \in y_{i} \rightarrow_{i} z_{i} \text { and any } i \in I \\
& \Leftrightarrow\left(x_{i}\right)_{i \in I} \leq\left(a_{i}\right)_{i \in I} \\
& \Leftrightarrow\left(x_{i}\right)_{i \in I} \ll\left(y_{i}\right)_{i \in I} \rightarrow\left(z_{i}\right)_{i \in I}, \text { since }\left(a_{i}\right)_{i \in I} \in\left(y_{i}\right)_{i \in I} \rightarrow\left(z_{i}\right)_{i \in I}
\end{aligned}
$$

So, we have proved the next theorem.

Theorem 3.8. If $\left\{\left(L_{i} ; \vee_{i}, \wedge_{i}, \odot_{i}, \rightarrow_{i}, 0_{i}, 1_{i}\right) \mid i \in I\right\}$ be a family of hyper residuated lattices, then

$$
\left(\Pi_{i \in I} L_{i} ; \vee, \wedge, \odot, \rightarrow,\left(0_{i}\right)_{i \in I},\left(1_{i}\right)_{i \in I}\right)
$$

is also a hyper residuated lattice.

Theorem 3.9. Let $\left(L_{1} ; \vee_{1}, \wedge_{1}, \odot_{1}, \rightarrow_{1}, 0_{1}, 1_{1}\right)$ and $\left(L_{2} ; \vee_{2}, \wedge_{2}, \odot_{2}, \rightarrow_{2}, 0_{2}, 1_{2}\right)$ be two hyper residuated lattices.

(i) If $F_{1}$ and $F_{2}$ are two (weak) filters of $L_{1}$ and $L_{2}$, respectively, then $F_{1} \times F_{2}$ is a (weak) filter of $L_{1} \times L_{2}$.

(ii) If $D_{1}$ and $D_{2}$ are two (weak) deductive systems of $L_{1}$ and $L_{2}$, respectively, then $D_{1} \times D_{2}$ is a (weak) deductive system of $L_{1} \times L_{2}$.

(iii) If $F$ is a (weak) filter of $L_{1} \times L_{2}$, then there exist two unique (weak) filters $F_{1}$ and $F_{2}$ of $L_{1}$ and $L_{2}$, respectively, such that $F=F_{1} \times F_{2}$.

(iv) If $D$ is a (weak) deductive system of $L_{1} \times L_{2}$, then there exist two (weak) deductive systems $D_{1}$ and $D_{2}$ of $L_{1}$ and $L_{2}$, respectively, such that $D=D_{1} \times D_{2}$. 
Proof. Suppose that $F_{1}$ and $F_{2}$ are filters of $L_{1}$ and $L_{2}$, respectively. Then $F_{1} \times F_{2} \neq \emptyset$. Let $(x, y) \in F_{1} \times F_{2}$ and $(x, y) \leq(a, b)$, for some $(a, b) \in L_{1} \times L_{2}$. Then $x \leq_{1} a$ and $y \leq_{2} b$ and so by (F), $a \in F_{1}$ and $b \in F_{2}$. Hence $(a, b) \in F_{1} \times F_{2}$. Now, let $(u, v),\left(u^{\prime}, v^{\prime}\right) \in F_{1} \times F_{2}$. Then $u, u^{\prime} \in F_{1}$ and $v, v^{\prime} \in F_{2}$. Since $F_{1}$ and $F_{2}$ are filters of $L_{1}$ and $L_{2}$, respectively, we get $u \odot_{1} u^{\prime} \subseteq F_{1}$ and $v \odot_{2} v^{\prime} \subseteq F_{2}$. Hence $(u, v) \odot\left(u^{\prime}, v^{\prime}\right) \subseteq F_{1} \times F_{2}$. Therefore, $F_{1} \times F_{2}$ is a filter of $L_{1} \times L_{2}$. The proof for weak filters is similar.

(ii) The proof is similar to the proof of (i).

(iii) Let $F$ be a (weak) filter of $L_{1} \times L_{2}, F_{1}=\left\{x \in L_{1} \mid(x, y) \in F\right.$, for some $\left.y \in L_{2}\right\}$ and $F_{2}=\left\{y \in L_{2} \mid(x, y) \in F\right.$, for some $\left.x \in L_{1}\right\}$. Since $F \neq \emptyset$, we have $F_{1} \neq \emptyset$ and $F_{2} \neq \emptyset$. Let $x \in F_{1}$ and $x \leq_{1} x^{\prime}$, for some $x^{\prime} \in L_{1}$. Then there exists $y \in L_{2}$ such that $(x, y) \in F$ and so by $(\mathrm{F}),(x, 1) \in F$. Since $(x, 1) \leq\left(x^{\prime}, 1\right)$ and $F$ is a (weak) filter of $L_{1} \times L_{2}$, we get that $\left(x^{\prime}, 1\right) \in F$ and so $x^{\prime} \in F_{1}$. Now, let $a, a^{\prime} \in F_{1}$. Then there exist $b, b^{\prime} \in L_{2}$ such that $(a, b),\left(a^{\prime}, b^{\prime}\right) \in F$. Hence $\left(a \odot_{1} a^{\prime}, b \odot_{2} b^{\prime}\right)=(a, b) \odot\left(a^{\prime}, b^{\prime}\right) \subseteq F\left(F \ll(a, b) \odot\left(a^{\prime}, b^{\prime}\right)=\left(a \odot_{1} a^{\prime}, b \odot_{2} b^{\prime}\right)\right)$ and so $a \odot_{1} a^{\prime} \subseteq F_{1}$ $\left(F \ll(u, v)\right.$, for some $u \in a \odot_{1} a^{\prime}$ and $v \in b \odot_{2} b^{\prime}$, which is implying $\left.a \odot_{1} a^{\prime} \cap F_{1} \neq \emptyset\right)$. Therefore, $F_{1}$ is a (weak) filter of $L_{1}$. By the similar way, we can prove that $F_{2}$ is a filter of $L_{2}$. Clearly, $F=F_{1} \times F_{2}$. Next, we show that $F_{1}$ and $F_{2}$ are unique. Let $H$ be a filter of $L_{1}$ and $K$ be a filter of $L_{2}$ such that $F=H \times K$. If $a \in H$, then $(a, 1) \in F$ and so $a \in F_{1}$. Conversely, if $a \in F_{1}$, then $(a, b) \in F$, for some $b \in L_{2}$ and so $a \in H$. Hence $H=F_{1}$. By the similar proof, we can show that $K=F_{2}$. Therefore, $F_{1}$ and $F_{2}$ are unique.

(iv) The proof is similar to the proof of (iii).

\section{The category of hyper residuated lattices}

In this section, we consider the category of hyper residuated lattices, whose object class is the class of all hyper residuated lattices and the morphisms are $S$-homomorphisms between them. We denote this category by $\mathcal{H} \mathcal{R} \mathcal{L}$ and study some categorical properties of this category in this section.

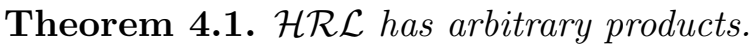

Proof. Let $\left\{\left(L_{i} ; \vee_{i}, \wedge_{i}, \odot_{i}, \rightarrow_{i}, 0_{i}, 1_{i}\right) \mid i \in I\right\}$ be an arbitrary family of hyper residuated lattices. We show that the hyper residuated lattice $\left(\Pi_{i \in I} L_{i} ; \vee, \wedge, \odot, \rightarrow,\left(0_{i}\right)_{i \in I},\left(1_{i}\right)_{i \in I}\right)$, which was defined in the last section, is a product of this family. Let $\pi_{i}: \Pi_{i \in I} L_{i} \rightarrow L_{i}$ be the canonical projection map, for any $i \in I$. Then clearly, for each $i \in I, \pi_{i}$ is a homomorphism. Let $(B ; \vee, \wedge, \odot, \rightarrow, 0,1)$ be a hyper residuated lattice and $\left\{\varphi_{i}: B \rightarrow L_{i} \mid i \in I\right\}$ be a family of the homomorphisms of $\mathcal{H} \mathcal{R}$ L. Define the map $\varphi: B \rightarrow \Pi_{i \in I} L_{i}$, by $\varphi(b)=(\varphi(b))_{i \in I}$, for each $b \in B$. It can be easily shown that the map $\varphi: B \rightarrow \Pi_{i \in I} L_{i}$ is the unique homomorphisms satisfying $\pi_{i} \circ \varphi=\varphi_{i}$. Therefore, $\left(\Pi_{i \in I} L_{i} ; \vee, \wedge, \odot, \rightarrow,\left(0_{i}\right)_{i \in I},\left(1_{i}\right)_{i \in I}\right)$ is the product of the family $\left\{\left(L_{i} ; \vee_{i}, \wedge_{i}, \odot_{i}, \rightarrow_{i}, 0_{i}, 1_{i}\right) \mid i \in\right.$ $I\}$.

Theorem 4.2. $\mathcal{H} \mathcal{R} \mathcal{L}$ has a terminal object.

Proof. Let $L=\{0\}$. Define the hyper operations $\vee, \wedge, \odot$ and $\rightarrow$ on $L$ by $0 \vee 0=0 \wedge 0=0 \odot 0=$ $0 \rightarrow 0=\{0\}$. Clearly, $(L, \vee, \wedge, \odot, \rightarrow, 0,0)$ is a hyper residuated lattice. It is easy to show that $L$ is a terminal object in $\mathcal{H} \mathcal{R} \mathcal{L}$. 
Definition 4.3. A non-empty subset $S$ of a hyper residuated lattice $L$ is called a subhyperlattice of $L$, if $S$ is closed with respect to $\vee, \wedge, \odot$ and $\rightarrow$. That is, for all $* \in\{\vee, \wedge, \odot, \rightarrow\}$ and $x, y \in S$, $x * y \subseteq S$. Then we say that $S$ with the same hyperoperations (restricted to $S$ ) is a subhyperlattice of $L$.

Proposition 4.4. Let $f: L \rightarrow L^{\prime}$ be a homomorphism, $S$ and $S^{\prime}$ be subhyperlattices of $L$ and $L^{\prime}$, respectively. Then $f(S)$ and $f^{-1}\left(S^{\prime}\right)$ are subhyperlattices of $L^{\prime}$ and $L$, respectively.

Proof. One can easily show the claim.

Theorem 4.5. Let $f: L \rightarrow L^{\prime}$ and $g: L \rightarrow L^{\prime}$ be two homomorphisms. Then $f$ and $g$ have an equalizer if and only if $A$, the set of all subhyperlattices of $L$ contained in $S=\{x \in L \mid f(x)=$ $g(x)\}$, has a maximum element (with respect to $\subseteq$ ).

Proof. $(\Rightarrow)$ Let $(e, E)$ be an equalizer of $f$ and $g$. By Proposition $4.4, e(E)$ is a subhyperlattice of $L$. Since $f(e(x))=g(e(x))$, for all $x \in E, e(E) \in A$. Clearly, if $\left\{A_{i} \mid i \in I\right\}$ is a chain of elements of $A$, then $\cup\left\{A_{i} \mid i \in I\right\}$ is an elements of $A$, too. Hence by Zorn's Lemma, $A$ has a maximal element. Let $M$ be a maximal element of $A$ and $i: M \rightarrow L$ be the inclusion map. Then $i$ is a morphism and $f \circ i=g \circ i$. Hence there exists a unique morphism $\phi: M \rightarrow E$ such that $e \circ \phi=i$ and so $M=i(M)=e(\phi(M)) \subseteq e(E)$. Since $M$ is a maximal element of $A$ and $e(E) \in A$, we have $e(E)=M$. Therefore, $e(E)=X$, for any maximal element $X$ of $A$ and so $A$ has maximum element.

$(\Leftarrow)$ Let $M$ be a maximum element of $A$ and $i: M \rightarrow L$ be the inclusion map. Then $f(i(x))=$ $g(i(x))$, for any $x \in M$. Now, let $(H, \vee, \wedge, \odot, \rightarrow, 0,1)$ be a hyper residuated lattice and $h: H \rightarrow L$ be a morphism such that $f \circ h=g \circ h$. Then by Proposition 4.4 $h(H) \in A$ and so $h(H) \subseteq M$. Hence $i \circ h=h$. Finally, if $h^{\prime}: H \rightarrow M$ is another morphism such that $i \circ h^{\prime}=h$, then clearly, $h^{\prime}=h$ and so $(i, M)$ is an equalizer of $f$ and $g$.

Theorem 4.6. Let $L_{1}$ and $L_{2}$ be two hyper residuated lattices, $f: L_{1} \rightarrow L$ and $g: L_{2} \rightarrow L$ be two homomorphisms and $S=\left\{(x, y) \in L_{1} \times L_{2} \mid f(x)=g(x)\right\}$. If $U$, the set of all subhyperlattices of $L_{1} \times L_{2}$ contained in $S$, has a maximum element, then the pullback of $f$ and $g$ exists.

Proof. Let $U$ has a maximum element. For any $i \in\{1,2\}$, define the maps $\alpha_{i}: M \rightarrow L_{i}$, by $\alpha_{i}\left(x_{1}, x_{2}\right)=x_{i}, i=1,2$. Clearly, $\alpha_{1}$ and $\alpha_{2}$ are two morphisms and $f \circ \alpha_{1}=g \circ \alpha_{2}$. Now, we show that $\left(\alpha_{1}, \alpha_{2}\right)$ is a pullback of $f$ and $g$. Let $\left(L_{3}, \vee, \wedge, \odot, \rightarrow, 0,1\right)$ be a hyper residuated lattice and $\beta_{i}: L_{3} \rightarrow L_{i}, i=1,2$ be two morphisms such that $f \circ \beta_{1}=g \circ \beta_{2}$. Then we define $\phi: L_{3} \rightarrow L_{1} \times L_{2}$, by $\phi(x)=\left(\beta_{1}(x), \beta_{2}(x)\right)$, for any $x \in L_{3}$. By Proposition $4.4, \phi\left(L_{3}\right) \in U$. Since $M$ is a maximal element of $U$, then $\phi\left(L_{3}\right) \subseteq M$ and so $\phi$ is a morphism from $L_{3}$ to $M$. It is easy to show that $\alpha_{1} \circ \phi=\alpha_{2} \circ \phi$. Now, let $\phi^{\prime}: L_{3} \rightarrow M$ be another morphism such that $\alpha_{1} \circ \phi^{\prime}=\alpha_{2} \circ \phi^{\prime}$. Then by definitions of $\alpha_{1}$ and $\alpha_{2}$ we get that $\phi=\phi^{\prime}$. Therefore, $\left(\alpha_{1}, \alpha_{2}\right)$ is the pullback of $f$ and $g$.

Now, we show that the category $\mathcal{H} \mathcal{R} \mathcal{L}$ does not have the initial objects and so it is not a cocomplete category.

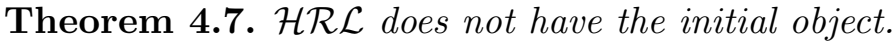

Proof. Let $M$ be an initial object in $\mathcal{H} \mathcal{R} \mathcal{L}$.

(i) If $|M|=1$, then $M$ is a trivial hyper residuated lattice. Let $L$ be a non-trivial hyper residuated lattice. If there exists a homomorphism $f: M \rightarrow L$, then $f(0)=0$ and so by $(P 1)$ we get

$$
1 \in f(0) \rightarrow f(0)=f(0 \rightarrow 0)=f(\{0\})=\{0\},
$$


which is a contradiction.

(ii) If $|M|=2$, then $M=\{0,1\}$. Let $L=\{0, a, 1\}$ be a partially ordered set such that $0<a<1$. For any $x, y \in L$, define $x \odot y=x \wedge y=\{\min \{x, y\}\}$ and $x \vee y=\{\max \{x, y\}$. Consider the following table:

Table 5

\begin{tabular}{|l|ccr|}
\hline$\rightarrow$ & 0 & a & 1 \\
\hline 0 & $\{1\}$ & $\{1\}$ & $\{1, \mathrm{a}\}$ \\
$\mathrm{a}$ & $\{0\}$ & $\{1\}$ & $\{1\}$ \\
1 & $\{0\}$ & $\{\mathrm{a}\}$ & $\{1\}$ \\
\hline
\end{tabular}

Then $(L ; \vee, \wedge, \odot, \rightarrow, 0,1)$ is a hyper residuated lattice. Hence there is a unique morphism $f: M \rightarrow$ $L$. But we have

$$
\{1, a\}=0 \rightarrow 1=f(0) \rightarrow f(1)=f(0 \rightarrow 1)=f(\{0,1\})=\{0,1\},
$$

which is a contradiction.

(iii) Finally, we assume that $3 \leq|M|$. Let $L=\{0, a, b, 1\}$ be a partially ordered set having the following Hasse diagram (Figure 3 ).

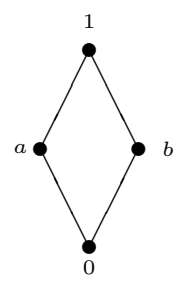

Figure 3:

Define the hyper operations $\vee, \wedge, \odot$ and $\rightarrow$ by $x \vee y=\{\max \{x, y\}\}, x \odot y=x \wedge y=\{\min \{x, y\}\}$. Now, consider the following diagram.

Table 6

\begin{tabular}{|l|cccr|}
\hline$\rightarrow$ & 0 & $\mathrm{a}$ & $\mathrm{b}$ & 1 \\
\hline 0 & $\{1\}$ & $\{1\}$ & $\{1\}$ & $\{1, \mathrm{a}, \mathrm{b}\}$ \\
$\mathrm{a}$ & $\{\mathrm{b}\}$ & $\{1\}$ & $\{\mathrm{b}\}$ & $\{1\}$ \\
$\mathrm{b}$ & $\{\mathrm{a}\}$ & $\{\mathrm{a}\}$ & $\{1\}$ & $\{1\}$ \\
1 & $\{0\}$ & $\{\mathrm{a}\}$ & $\{\mathrm{b}\}$ & $\{1\}$ \\
\hline
\end{tabular}

Since $M$ is an initial object, there exists a unique homomorphism $f: M \rightarrow L$. We claim that $f(x) \in\{a, b\}$, for some $x \in M$. If $f(M) \subseteq\{0,1\}$, then

$$
\{a, b, 1\}=0 \rightarrow 1=f(0) \rightarrow f(1)=f(0 \rightarrow 1) \subseteq f(M) \subseteq\{0,1\},
$$

which is a contradiction. Hence there exists $x \in M$ such that $f(x) \in\{a, b\}$. Define $f^{\prime}: M \rightarrow L$, by

$$
f^{\prime}(x)= \begin{cases}f(x) & \text { if } f(x) \in L-\{a, b\} \\ b & \text { if } f(x)=a, \\ a & \text { if } f(x)=b .\end{cases}
$$

It is not difficult to show that $f^{\prime}: M \rightarrow L$ is a morphism. Clearly $f \neq f^{\prime}$, which is a contradiction.

From (i), (ii) and (iii) we get that $\mathcal{H} \mathcal{R} \mathcal{L}$ does not have the initial objects. 
Corollary 4.8. The category $\mathcal{H} \mathcal{R} \mathcal{L}$ is not cocomplete.

Proof. It follows from Theorem 4.7 .

Proposition 4.9. Let $L$ be a hyper residuated lattice such that 1 is scalar and $f: L \rightarrow L^{\prime}$ is a homomorphism. Then $f$ is a one to one homomorphism if and only if $f^{-1}(1)=\{1\}$.

Proof. Let $f$ is a homomorphism such that $f^{-1}(1)=\{1\}$. If $f(x)=f(y)$, then $1 \in(f(x) \rightarrow$ $f(y)) \cap(f(y) \rightarrow f(x))$ and so $1 \in f(x \rightarrow y) \cap f(y \rightarrow x)$. Hence $((x \rightarrow y) \cap(y \rightarrow x)) \cap f^{-1}(1) \neq \emptyset$ and so $1 \ll x \rightarrow y$ and $1 \ll y \rightarrow x$. Now, by assumption, we have $\{x\}=1 \odot x \ll y$ and $y=1 \odot y \ll x$ and so $x=y$. Hence $f$ is a one to one homomorphism. The proof of the converse is straightforward.

Definition 4.10. A hyper residuated lattice $L$ is a subdirect product of an indexed family $\left\{L_{i}\right\}_{i \in I}$ of hyper residuated lattices if

- $L$ is a subhyperlattice of $\Pi_{i \in I} L_{i}$,

- $\pi_{i}(L)=L_{i}$, for any $i \in I$.

A one to one homomorphism $\alpha: L \rightarrow \prod_{i \in I} L_{i}$ is called subdirect embedding if $\alpha(L)$ is a subdirect product of the family $\left\{L_{i}\right\}_{i \in I}$. A hyper residuated lattice $L$ is called subdirectly irreducible if for every subdirect embedding $\alpha: L \rightarrow \prod_{i \in I} L_{i}$ there exists $i \in I$ such that $\pi_{i} \circ \alpha: L \rightarrow L_{i}$ is an S-isomorphism.

Let $(P, \leq)$ be a partially ordered set. Then we define $\downarrow x:=\{u \in P \mid u \leq x\}$ and $\uparrow x:=\{u \in$ $P \mid x \leq u\}$, for any $x \in P$.

Theorem 4.11. Let $\Delta=\{(x, x) \mid x \in L\}$ and $\left\{\theta_{i} \mid i \in I\right\}$ be the set of all regular compatible congruence relations of $L$ which are not equal to $\Delta$.

(i) If $L$ is subdirectly irreducible, then $L=\{0,1\}$ or $\bigcap\left\{\theta_{i} \mid i \in I\right\} \neq \Delta$.

(ii) If $x \wedge y \subseteq \downarrow x$ or $x \vee y \subseteq \uparrow x$, for any $x, y \in L$, then the converse of (i) is also true.

Proof. Let $L$ be subdirect irreducible, $L \neq\{0,1\}$ and $\Delta=\bigcap\left\{\theta_{i} \mid i \in I\right\}$. By Theorem 2.11, $L / \theta_{i}$ is a hyper residuated lattice, for any $i \in I$. Define the map $\alpha: L \rightarrow \prod_{i \in I} L / \theta_{i}$, by $\alpha(x)=\left([x]_{\theta_{i}}\right)_{i \in I}$. It can be easily obtain that $\alpha$ is an homomorphism and $\pi_{i}(\alpha(L))=L / \theta_{i}$, for any $i \in I$. Let $\alpha(x)=\alpha(y)$, for some $x, y \in L$. Then $\left([x]_{\theta_{i}}\right)_{i \in I}=\left([y]_{\theta_{i}}\right)_{i \in I}$ and so $(x, y) \in \theta_{i}$, for any $i \in I$. Since $\bigcap\left\{\theta_{i} \mid i \in I\right\}=\Delta$, we get $(x, y) \in \Delta$, and so $x=y$. Hence $\alpha$ is a subdirect embedding. Since $L$ is subdirect irreducible, there exists $j \in I$ such that $\phi_{j} \circ \alpha: L \rightarrow L / \theta_{j}$ is an isomorphism. It follows that $\theta_{j}=\Delta$, which is a contradiction. Therefore, $\bigcap\left\{\theta_{i} \mid i \in I\right\} \neq \Delta$.

(ii) Let $L=\{0,1\}$ and $\alpha: L \rightarrow \prod_{i \in I} L_{i}$ be a subdirect embedding. Then $\pi_{i}(\alpha(L))=L_{i}$, for any $i \in I$. Since $\alpha$ is a one to one homomorphism, we get that $\alpha(1) \neq \alpha(0)$ and so there exists $i \in I$ such that $0_{i} \neq 1_{i}$. By $\alpha(0)=\left(0_{i}\right)_{i \in I}$ and $\alpha(1)=\left(1_{i}\right)_{i \in I}$, we get $\pi_{i}(\alpha(0))=0_{i}$ and $\pi_{i}(\alpha(1))=1_{i}$ and so $\pi_{i} \circ \alpha: L \rightarrow L_{i}$ is one to one. Hence $\pi_{i} \circ \alpha: L \rightarrow L_{i}$ is an $S$-isomorphism and so $L$ is subdirect irreducible. Now, let $\bigcap\left\{\theta_{i} \mid i \in I\right\} \neq \Delta$. We will show that $L$ is subdirect irreducible. Let $\left\{L_{i}\right\}_{i \in J}$ be a family of hyper residuated algebras and $\pi_{i}: L \rightarrow \prod_{i \in J} L_{i}$ be a subdirect embedding. Let $\beta_{i}=\operatorname{ker}\left(\pi_{i} \circ \alpha\right)$, for any $i \in J$. If $\pi_{i} \circ \alpha(x) \leq \pi_{i} \circ \alpha(y)$, for some $x, y \in L$, then $\pi_{i} \circ \alpha(x) \in \pi_{i} \circ \alpha(x) \pi \pi_{i} \circ \alpha(y)$. Since $\pi_{i} \circ \alpha$ is a homomorphism, we obtain $\pi_{i} \circ \alpha(x) \in \pi_{i} \circ \alpha(x \wedge y)$ and so there exists $a \in x \wedge y$ such that $(x, a) \in \operatorname{ker}\left(\pi_{i} \circ \alpha\right)$. By the similar 
way, we can show that, there exists $b \in x \vee y$ such that $(y, b) \in \operatorname{ker}\left(\pi_{i} \circ \alpha\right)$. Now, by assumption $x \wedge y \subseteq \downarrow y$ or $x \vee y \subseteq \uparrow x$. If $x \wedge y \subseteq \downarrow y$, then $a \leq y$ and so $\{x, x, a, y\}$ forms a $\beta_{i}$-fence that joins $x$ to $y$ (see Figure 4(i)). If $x \vee y \subseteq \uparrow x$, then the set $\{x, b, y, y\}$ forms a $\beta_{i}$-fence that joins $x$ to $y$ (see Figure 4(ii)). Hence by Theorem 2.12, $\beta_{i}$ is a regular compatible congruence relations of $L$, for any $i \in J$. Clearly, $\Delta=\operatorname{ker}(\alpha)=\bigcap\left\{\beta_{i} \mid i \in J\right\}$. Since $\bigcap\left\{\theta_{i} \mid i \in I\right\} \neq \Delta$, we conclude that there exists $j \in J$ such that $\beta_{j}=\Delta$ and so $\pi_{j} \circ \alpha: L \rightarrow L_{j}$ is an isomorphism. Therefore, $L$ is subdirect irreducible.

(i)

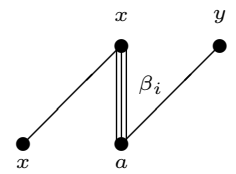

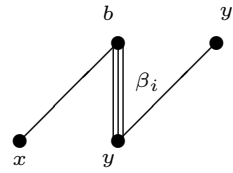

(ii)

Figure 4:

Now, we attempt to construct a hyper residuated lattice from a residuated lattice. A hyper residuated lattice is called a normal hyper residuated lattice if $(L, \vee, \wedge, 0,1)$ is a bounded lattice. In the following, we want to verify the relation between the category of residuated lattices and the category whose object class is the set of all normal hyper residuated lattices and the morphisms are homomorphism between them.

Theorem 4.12. Let $(L, \vee, \wedge, \odot, \rightarrow, 0,1)$ be a residuated lattice. For any $x, y \in L$, we define $x \rightsquigarrow y=\{u \in L \mid u \leq x \rightarrow y\}$ and $x \circ y=\{u \in L \mid x \odot y \leq u\}$. Then $(L, \vee, \wedge, \circ, \rightsquigarrow, 0,1)$ is a hyper residuated lattice.

Proof. It suffices to show that $(L, \circ, 1)$ is a commutative semihypergroup and also $x \circ y \ll z$ if and only if $x \ll y \rightsquigarrow z$, for any $x, y, z \in L$. Since $(L, \vee, \wedge, \odot, \rightarrow, 0,1)$ is a residuated lattice, $x \odot 1=x$ and $x \odot y=y \odot x$, for all $x, y, \in L$. This gives $x \in x \circ 1$ and $x \circ y=y \circ x$. Now, let $u \in(x \circ y) \circ z$, then there exists $a \in x \circ y$ such that $u \in a \circ z$. Hence $a \odot z \leq u$. Further, $a \in x \circ y$ gives $x \odot y \leq a$. Hence by $(R 2),(x \odot y) \odot z \leq a \odot z \leq u$ and so $u \in\{v \in L \mid(x \odot y) \odot z \leq v\}$. Moreover, $a \odot b \in a \circ b$ and $s \in a \circ b$ imply $\uparrow s \subseteq a \circ b$, for any $a, b \in L$. Hence $(x \odot y) \odot z \in(x \circ y) \circ z$. It follows that, $\{v \in L \mid(x \odot y) \odot z \leq v\} \subseteq(x \circ y) \circ z$. Therefore, $\{v \in L \mid(x \odot y) \odot z \leq v\}=(x \circ y) \circ z$. By the similar way, we can show that $\{v \in L \mid x \odot(y \odot z) \leq v\}=x \circ(y \circ z)$. Since $(L, \odot, 1)$ is a commutative monoid, $(x \circ y) \circ z=x \circ(y \circ z)$ and so $(L, \circ, 1)$ is a commutative semihypergroup. Now, let $x \circ y \ll z$. Then there exists $a \in x \circ y$ such that $a \leq z$. Since $x \odot y \leq a$, we get $x \odot y \leq z$ and so $x \leq y \rightarrow z$. Hence $x \in y \rightsquigarrow z$, whence $x \ll y \rightsquigarrow z$. Conversely, if $x \ll y \rightsquigarrow z$, then there exists $a \in y \rightsquigarrow z$ such that $x \leq a$. Since $a \leq y \rightarrow z$, we get $a \leq y \rightarrow z$ and so $a \odot y \leq z$. Since $x \leq a$, by $(R 2), x \odot y \leq a \odot y \leq z$ and so $x \circ y \ll z$. Hence $x \circ y \ll y$ if and only if $x \ll y \rightsquigarrow z$. Therefore, $(L, \vee, \wedge, \circ, \rightsquigarrow, 0,1)$ is a hyper residuated lattice.

If $(L, \vee, \wedge, \odot, \rightarrow, 0,1)$ be a residuated lattice, then we use $F(L)$ to denote the hyper residuated lattice induced from $L$ in Theorem 4.12 .

Theorem 4.13. Let $(L, \vee, \wedge, \odot, \rightarrow, 0,1)$ and $\left(L^{\prime}, \vee, \wedge, \odot, \rightarrow, 0,1\right)$ be two residuated lattices and $f: L \rightarrow L^{\prime}$ be a homomorphism. We define $F(f): F(L) \rightarrow F\left(L^{\prime}\right)$, by $F(f)(x)=f(x)$, and $F(f)(A)=\{f(a) \mid a \in A\}$, for any $x \in L$ and $A \subseteq L$. Then

(i) $F(f): F(L) \rightarrow F\left(L^{\prime}\right)$ is a homomorphism,

(ii) $F$ is a covariant functor from the category of residuated lattices to the category of normal hyper residuated lattices. 
Proof. (i) By the definition of $F(L)$ and $F\left(L^{\prime}\right)$, it suffices to show that $F(f)(x \rightsquigarrow y) \subseteq F(f)(x) \rightsquigarrow$ $F(f)(y)$ and $F(f)(x \circ y) \subseteq F(f)(x) \circ F(f)(y)$, for any $x, y \in L$. Let $x, y \in L$. If $a \in F(f)(x \rightsquigarrow y)$, then there exists $u \in x \rightsquigarrow y$ such that $a=f(u)$. Hence $u \odot x \leq y$. Since $f$ is a homomorphism, we get $a \odot f(x)=f(u) \odot f(x)=f(u \odot x) \leq f(y)$ and so $a \leq f(x) \rightarrow f(y)$. Thus, $a \in f(x) \rightsquigarrow f(y)$. That is, $F(f)(x \rightsquigarrow y) \subseteq F(f)(x) \rightsquigarrow F(f)(y)$. Moreover,

$$
F(f)(x \circ y)=f(x \circ y)=f(\{u \in L \mid x \odot y \leq u\})=\{f(u) \mid u \in L, \quad x \odot y \leq u\} .
$$

Let $a \in F(f)(x \circ y)$. Then there exists $u \in L$ such that $a=f(u)$ and $u \leq x \rightarrow y$. Since $f$ is a homomorphism, we get $a=f(u) \leq f(x \rightarrow y)=f(x) \rightarrow f(y)$ and so $a \in f(x) \rightsquigarrow f(y)$. Hence $F(f)(x y) \subseteq F(f)(x) \rightsquigarrow F(f)(y)$. Therefore, $F(f)$ is a homomorphism.

(ii) Let $L, L^{\prime}$ and $L^{\prime \prime}$ be three residuated lattices and $f: L \rightarrow L^{\prime}, g: L^{\prime} \rightarrow L^{\prime \prime}$ be two homomorphisms. Then by definition of $F$, clearly $F\left(I d_{L}\right)=I d_{F(L)}$. Hence by $F(g \circ f)(x)=g(f(x))=$ $(F(g) \circ F(f))(x)$, for any $x \in L$, we conclude that $F$ is a covariant functor from the category of residuated latices to the category of normal hyper residuated lattices.

\section{Conclusions}

In this paper, we introduced and studied category of hyper residuated lattices. First, we introduced homomorphisms (S-homomorphism) between hyper residuated lattices and found their properties. Then we consider the category whose objects are hyper residuated lattices and whose morphisms are S-homomorphism, We showed that, $\mathcal{H} \mathcal{R} \mathcal{L}$ has a terminal object and arbitrary products, but it does not have the initial object (consequently, it is not cocomplete). Also, we found a condition which under the pullback of S-homomorphisms $f$ and $g$ exists (Theorem 4.6). Finally, we introduced a subcategory of $\mathcal{H} \mathcal{R} \mathcal{L}$ entitled, normal hyper residuated lattice, and found a covariant functor from the category of residuated lattices to this subcategory (Theorem 4.13).

\section{References}

[1] S. Awodey, Category theory, Oxford University Press, United States, (2006).

[2] T.S. Blyth, Lattices and ordered algebraic structures, Springer-Verlag, (2005).

[3] R. Belohlavek, Some properties of residuated lattices, Czechoslovak Mathematical Journal, 53 (2003), 161-171.

[4] R.A. Borzooei, M. Bakhshi, O. Zahiri, Filter theory on hyper residuated lattices, Quasigroups and Related Systems, 22(1) (2014), 33-50.

[5] R.A. Boorzooei, A. Hasankhani, M.M. Zahedi, Y.B. Jun, On hyper K-algebra, Mathematica Japonica, 1 (2000), 113-121.

[6] C.C. Chang, Algebraic analysis of many valued logics, Transactions of the American Mathematical Society, 88 (1958), 467-490.

[7] P. Corsini, Prolegomena of hypergroup, Aviani Editore, (1993).

[8] P. Corsini, V. Leoreanu, Applications of hyperstructure theory, Kluwer Academic Publishers, Dordrecht, (2003). 
[9] F. Esteva, L. Godo, Monoidal t-norm based logic: Towards a logic for left-continuous t-norms, Fuzzy Sets and Systems, 124, (2001), 271-288.

[10] Sh. Ghorbani, A. Hasankhani, E. Eslami, Hyper MV-algebras, Set-Valued Mathematics and Applications, 1 (2008), 205-222.

[11] P. Hájek, Metamathematics of fuzzy logic, Kluwer Academic Publishers, Dordrecht, (1998).

[12] J. Mittas, M. Konstantinidou, Sur une nouvelle génération de la notion de treillis. Les supertreillis et certaines de leurs proprités générales, Annales Mathmatiques Blaise Pascal (Clermont II), Sér. Math. Fasc., 25 (1989), 61-83.

[13] M. Ward, R.P. Dilworth, Residuated lattices, Transactions of the American Mathematical Society, 45 (1939), 335-354.

[14] O. Zahiri, R.A. Borzooei, M. Bakhshi, (Quotient) hyper residuated lattices, Quasigroups and Related Systems, 20 (2012), 125-138. 